\title{
Spatial estimation: A non-Bayesian alternative
}

\author{
Hilary Barth ${ }^{1}$, Ellen Lesser ${ }^{1}$, Jessica Taggart ${ }^{1}$, \& Emily Slusser ${ }^{1,2}$ \\ ${ }^{1}$ Wesleyan University, ${ }^{2}$ San Jose State University
}

Keywords: spatial cognition, estimation, proportional reasoning, Bayesian

\section{Word Count: 3998}

Corresponding author: Hilary Barth, Department of Psychology, Wesleyan University, 207 High St, Middletown, CT 06459, hbarth@wesleyan.edu.

Author Note: This work was supported in part by NSF DRL-0950252 to HB and a Wesleyan University Psychology Department Postdoctoral Fellowship to ES. We thank Isabel Bernstein, Rachel Santiago, and Amy Toig for assistance with data collection, and the participating students and families who made this work possible. 
Spatial estimation: A non-Bayesian alternative

\begin{abstract}
A large collection of estimation phenomena (e.g., biases arising when adults or children estimate remembered locations of objects in bounded spaces; Huttenlocher, Newcombe, \& Sandberg, 1994) are commonly explained in terms of complex Bayesian models. We provide evidence that some of these phenomena may be modeled instead by a simpler non-Bayesian alternative. Undergraduates and 9-to-10-year-olds completed a speeded linear position estimation task. Bias in both groups' estimates could be explained in terms of a simple psychophysical model of proportion estimation. Moreover, some individual data were not compatible with the requirements of the more complex Bayesian model.
\end{abstract}


Spatial estimation: A non-Bayesian alternative

In recent years, Bayesian models of cognition have received considerable attention. Despite the appealing qualities and contributions of Bayesian modeling methods, critics argue that these models are sometimes highly flexible and complex (thus sacrificing predictive value), with excessive ad hoc specification in response to the very datasets they attempt to explain (e.g. Bowers \& Davis, 2012; Friedman, Montello, \& Burte, 2012; Jones \& Love, 2011; Marcus \& Davis, 2013; but see Griffiths, Chater, Norris, \& Pouget, 2012). With the current prevalence of Bayesian accounts, traditional models of many phenomena may be less frequently considered, even though they sometimes provide a good or better account of behavior (Bowers \& Davis, 2012; Jones \& Love, 2011; Mozer, Pashler, \& Homaei, 2008). To the extent that Bayesian models serve as computational-level descriptions of behavior rather than models of cognitive processing, they are not necessarily in competition with other accounts (e.g. Griffiths et al., 2012). However, Bayesian models are often presented as descriptions of cognitive processing. Here, we present a family of phenomena that have been explained most prominently in terms of relatively complex Bayesian processes. We show that a simpler non-Bayesian alternative can provide an excellent quantitative explanation of these phenomena.

When we estimate, our estimates are often biased. This is true across a wide variety of contexts. Whether adults are asked to make estimates of the magnitude of a grey square's lightness or the magnitude of a schematic fish's width (Huttenlocher, Hedges, \& Vevea, 2000), the position of a marked location within a circle (Huttenlocher, Hedges, \& Duncan, 1991), or the position of a number on a number line (Siegler \& Opfer, 2003; Cohen \& Blanc-Goldhammer, 2011; Sullivan, Juhasz, Slattery, \& Barth, 2011), systematic biases appear in their estimation patterns. Developmental researchers working within diverse theoretical frameworks have 
identified similar patterns in children completing estimation tasks (e.g. Barth \& Paladino, 2011;

Duffy, Huttenlocher, \& Crawford, 2006; Huttenlocher, Newcombe, \& Sandberg, 1994; Siegler \& Booth, 2004; Slusser, Santiago, \& Barth, 2013).

Here we focus on a simple family of estimation tasks in the spatial domain. People exhibit systematic bias when asked to reproduce a remembered location within a bounded linear space, such as identifying a previously observed position in a rectangular space or finding an object buried in a long thin sandbox (Huttenlocher et al., 1994). Huttenlocher and colleagues (1994) used these tasks to demonstrate that even one-year-olds can use distance information to code location, contrary to Piagetian theory. Their work also revealed suggestive patterns of bias in spatial position estimates, with characteristic developmental changes. Generally, adults' and older children's group estimates for locations within each half of the rectangular space are displaced toward the center of that half. In younger children, group estimates are displaced toward the center of the entire rectangular space. The degree of displacement varies in different regions of the space (see Figure 1).

These and related performance patterns are commonly explained in terms of a "Category Adjustment" (CA) model of spatial coding. ${ }^{1}$ The location is hierarchically coded at two levels: an inexact but unbiased fine-grained location, and a spatial category associated with the location (for example, a geometrically defined area surrounding the location). Estimates result from a Bayesian combination of the inexact fine-grained information about a stimulus location and information about the spatial category of which it is a member ${ }^{2}$. Category-level knowledge serves as prior information, combining in a weighted fashion with inexact stimulus values. Bias

\footnotetext{
${ }^{1}$ For a review of the rich literature on a substantially different approach, the dynamic field theory of spatial memory, see Spencer, Austin, \& Schutte, 2012; Schutte, Spencer, \& Schoner, 2003. 2 For theoretically related work in the domain of temporal interval reproduction, see Jazayeri \& Shadlen, 2010; Cicchini et al., 2012; Aagten-Murphy et al., 2013.
} 
originates from the adjustment of estimates towards the category prototype (usually the center of mass of the spatial category). This model explains the data mentioned above as follows: adults and older children subdivide the space into halves, with estimates displaced toward the center of each half, while younger children's estimates are displaced toward the center of the entire space because they haven't yet begun to subdivide. Multiple factors determine the degree of adjustment in the CA model; the key point is that this adjustment results in biased estimates. This is considered rational because, although it introduces bias, it also reduces estimation variability (Duffy et al., 2006; Huttenlocher et al., 2000).

The Category Adjustment model comprises a broad theory of the role of categories in cognition. It has been invoked to explain results from a host of estimation tasks, ranging from those involving geometric spatial categories to those involving inductive categories, across many domains in children and adults. These tasks include remembering physical locations in the real world (Holden, Newcombe, \& Shipley, 2013), recalling the location of various points within a photograph (Holden, Curby, Newcombe, \& Shipley, 2010), reporting the location of a dot in a circle (Huttenlocher et al., 1991), or reproducing the size of fish-shaped stimuli (Duffy et al., 2006; Huttenlocher et al., 2000). For example, when kindergarteners saw an image of a fishshaped stimulus and reproduced its size using a new adjustable fish, responses showed bias broadly consistent with the CA model (Duffy et al., 2006), purportedly showing that children use category information to improve estimation accuracy.

We suggest that conceptualizing these tasks differently may lead to a simpler explanation. Many tasks thought to provide evidence for the CA model could be conceptualized as proportion estimation tasks, in which participants estimate the magnitude of some part, not just in isolation, but in relation to the whole. They require estimation of particular stimulus 
values within a bounded range, which may be thought of as estimates of proportions of an explicitly or implicitly specified whole value. For example, to remember the location of an item in a long thin space with no landmarks, one must encode not simply an absolute distance but rather a distance relative to at least one reference point, such as one end of the space. One could potentially encode not simply an absolute distance, but rather the magnitude of the part (distance from endpoint to target location) and the magnitude of the whole (the distance between endpoints). Absolute encoding of a distance from an endpoint would be unhelpful if the position of the observer or the space changed, whereas proportional encoding (Huttenlocher, Newcombe, \& Vasilyeva, 1999) would have the advantage of scaling across situations and viewing distances (consistent with research on children's spatial scaling, e.g. Frick \& Newcombe, 2012, and extent encoding, e.g. Duffy, Huttenlocher, \& Levine, 2005).

It is widely acknowledged that estimates of proportions and frequencies are biased such that smaller proportions are overestimated and larger ones are underestimated (for reviews, see Hollands \& Dyre, 2000; Zhang \& Maloney, 2012). This finding has been reproduced in hundreds if not thousands of studies (e.g. judging the proportion of black elements in an array of black and white elements, Varey, Mellers, \& Birnbaum, 1990; judging the percentage of a letter in a mixed sequence, Erlick, 1964; and judging the ratio of durations of short time intervals, Nakajima, 1987). Because these patterns of bias in proportion estimation are qualitatively similar to those arising from tasks used to explore the CA model, and CA tasks can also be conceptualized as proportion estimation tasks, it is possible that theoretical explanations of proportion estimation may be extended to tasks from the CA literature.

Hollands \& Dyre (2000) proposed a model based in the psychophysical tradition that accounts for estimation biases resulting from a wide variety of perceptually based tasks, each of 
which involves explicit or implicit estimates of proportion (the Cyclical Power Model of proportion estimation or CPM, Hollands \& Dyre, 2000). Bias in the estimation of positions within 2D shapes (Huttenlocher et al.,1991) can be explained in terms of such a model, as can many other examples of bias in perceptual estimation (Hollands \& Dyre, 2000). This model has also been invoked recently as an explanation of estimation bias for proportions specified by symbols representing abstract quantities, as in the case of number-line estimation (Barth \& Paladino, 2011; Cohen \& Blanc-Goldhammer, 2011; Cohen \& Sarnecka, 2014; Rouder \& Geary, in press; Slusser et al., 2013; Sullivan et al., 2011), and it is qualitatively compatible with a larger set of phenomena that remain to be tested quantitatively.

This account is based on the idea that bias in proportion estimation originates from bias involved in the estimation of each part magnitude, as "psychological" magnitude often does not equal true, physical magnitude. Spence (1990) showed that S-shaped and reverse S-shaped patterns of bias in observers' estimates of the proportional sizes of various shapes on a graph could be explained by a psychophysical model of proportion estimation. The model is based on Stevens' Law, which describes the relationship between the estimated or perceived magnitude of a stimulus and its actual magnitude as a power function $y=\alpha x^{\beta}$ (the exponent $\beta$ quantifies bias associated with judgments of a particular stimulus continuum ${ }^{3}$, e.g., brightness or area, and $\alpha$ is a scaling parameter). Estimates of proportions of magnitudes take the form of S-shaped or reverse S-shaped curves (see Figure $2 \mathrm{~A}$ ), with the value of $\beta$ determining degree and direction of curvature (Spence, 1990). When observers respond based on two bounding reference points in their judgments of proportion, estimates are predicted by $y=x^{\beta} /\left(x^{\beta}+(1-x)^{\beta}\right)$. This model (Figure 2A) would apply, for example, if an observer estimated the liquid-filled proportion of a

3 See Teghtsoonian, 2012, for a recent overview. 
cylinder by judging the liquid's level relative to the entire height of the cylinder. This simple model was later generalized (Hollands \& Dyre, 2000) to cases in which additional reference points are used (e.g., estimating the filled proportion of the cylinder by judging the liquid's level relative to half the height of the cylinder). Using additional reference points in this manner produces multiple S-shaped or reverse S-shaped cyclical estimation patterns (Figure 2B). It also increases overall estimation accuracy, even when values of $\beta$ remain constant. Such cycles are observed in children's number-line estimates, with developmental change arising in part from the increasing use of additional reference points with age (Barth \& Paladino, 2011; Rouder \& Geary, in press; see Slusser et al., 2013, for details). Cyclical patterns also arise in proportion estimates using various perceptual continua in adults (Hollands \& Dyre, 2000), and closely resemble the patterns of estimation bias arising in many tasks from the CA literature (Figure 1).

Thus, estimation biases thought to support the CA model are similar to those that have been explained in terms of a proportion estimation model in different tasks. Although the linear position estimation tasks described earlier can also be conceived as proportion estimation tasks, the CPM has not been applied to these tasks to date. Here we focus on these spatial estimation tasks to ask whether this relatively simple non-Bayesian model can explain the data. Because CA-based accounts of estimation bias have been proposed for both adults and children, we asked whether a proportion estimation model could similarly explain performance in both adults and children.

We examined estimation biases evoked by a spatial position estimation task in adults and in children aged 9-10. Participants observed a horizontal line with a vertical hatchmark, and made a speeded mouseclick to reproduce that hatchmark's location on a new unmarked line in a novel position. We asked, for each group, whether psychophysical models of proportion 
estimation could explain the estimation data and provide a simpler alternative to the more complex Bayesian Category Adjustment Model.

\section{Method}

\section{Participants}

Participants were 46 children (recruited from a local participant database) and 49 undergraduate students (participating for pay or course credit). Twelve participants were excluded for being outside the target age range, expressed developmental delay or concussion, non-completion, computer error, or missed instructions. Of the participants contributing data, 20 were 9-year-olds (mean age 9;6), 20 were 10-year-olds (mean age 10;4) and 43 were adults. Testing took place in a quiet laboratory room.

\section{Stimuli}

Stimuli were created using MATLAB and displayed in a pseudorandom order for each participant. For each trial, a centered fixation rectangle (grey, $12.3 \mathrm{~cm}$ x $0.7 \mathrm{~cm}$ ) was immediately followed by a stimulus screen and a response screen. Fixation rectangles and stimulus screens each lasted $500 \mathrm{~ms}$ for adults and $750 \mathrm{~ms}$ for children. Response screens were displayed for $1500 \mathrm{~ms}$ for adults and $2250 \mathrm{~ms}$ for children. The stimulus screen displayed a centered $12.3 \mathrm{~cm}$ horizontal line with unlabeled "endpoints" (short vertical lines at each end extending $0.3 \mathrm{~cm}$ above and below the line) and a vertical hatch mark at a target position. The spatial response screen presented a non-centered pseudorandomly located line of identical size and orientation, with no hatch mark. For the purposes of assigning target positions, the endpoints of the line were assigned values of 0 and 1000 (these labels were not presented to the participants). Target values to be estimated were sampled at intervals of approximately 50 units 
(a uniform distribution), with the exact presented values jittered slightly (e.g. positions corresponding to " 47 " and " 51 " were presented rather than two instances of " 50 "). Target positions used were those corresponding to the numbers $47,51,98,102,147,153,199,202,249$, $252,298,302,349,351,398,403,449,453,499,502,547,552,597,601,647,652,699,703$, 747, 753, 798, 802, 848, 853, 899, 901, 949, and 953.

\section{Design}

Participants completed the spatial estimation task and a number-line estimation task. Participants completed two number-line $(\mathrm{N})$ trial blocks and two spatial estimation $(\mathrm{S})$ trial blocks. Adults completed 38 trials/block (each target value represented twice) in NSSN or SNNS order. Children completed 19 trials/block (each target value represented once), with blocks in NNSS or SSNN order to reduce the number of potentially confusing task switches. The numberline task was part of a separate study and will not be discussed further here.

\section{Procedure}

Participants were seated in front of a computer with blank paper covering the keyboard and top of the screen to obscure potential landmarks. Adults were given written and spoken instructions for the first block; children received spoken instructions and examples on paper. Four practice trials (for children) or two practice trials (for adults) preceded the first block. For each trial, the participant's task was to move the cursor and click the appropriate position (the position that matched the previously presented spatial location) on the blank horizontal line during the response screen. Mouseclicks were recorded as numbers from 0-1000, corresponding to locations along the response line. A $1000 \mathrm{~ms}$ pause separated trials for adults and a $1500 \mathrm{~ms}$ pause separated trials for children. For children, the experimenter also had the option to pause the game in case of distraction. 


\section{Results and Discussion}

Participants' estimates were compared to the actual locations corresponding to the target spatial positions. All individuals produced estimates correlated with the target positions in each block, showing that they understood the instructions and were motivated to complete the task. An individual's estimate for a target value was removed as an outlier if it differed from the group mean for that target value by more than 2 SDs $(3.72 \%$ of trials for children, $3.10 \%$ of trials for adults). The median estimates of the 9-and 10-year-olds did not differ, $t(74)=-0.04, p>0.05$; for further analyses, the two age groups were combined into one group of children. Percent absolute error (PAE) was calculated as a general measure of accuracy (see Booth \& Siegler, 2006, 2008; Slusser et al., 2013). Though both groups were extremely accurate (adults: PAE = $2.5 \%$; children: $\mathrm{PAE}=3.0 \%$ ), systematic estimation biases did arise.

Figure 3 depicts the median estimates of the adults and the children. Data were fitted with psychophysical models of proportion estimation (Hollands \& Dyre, 2000; Slusser et al., 2013; Spence, 1990). These models (see Figure 2A, 2B) account for cyclical over- and underestimation patterns such as those seen in the present data. Their one free parameter, $\beta$, corresponds to the exponent in Stevens' Power Law; this parameter gives the degree and direction of bias (how the lines curve away from the $\mathrm{X}$-axis in Figure 2), with values over 1 corresponding to estimates being shifted rightward on the left half of the line and leftward on the right half and values under 1 corresponding to the opposite pattern. Single-cycle (Fig. 2A) or multiple-cycle (Fig. 2B) versions of the model account for cases in which participants judge quantities as proportions of a single whole vs. cases in which participants use additional reference points. 
We fitted one- and two-cycle versions of the model and found that the two-cycle version provided the better explanation of the data for both groups (see Figure 3), yielding lower AICc scores (for adults, $\Delta \mathrm{AICc}=15.95$; for children, $\Delta \mathrm{AICc}=17.38)^{4}$. The resulting value of $\beta$ was between 0 and 1 for both groups, showing that the direction of bias was the same (on average) for both. The degree of bias was also nearly identical for both groups (adults: $\beta=0.8130, R^{2}=$ 0.9964; children: $\left.\beta=0.8174, \mathrm{R}^{2}=0.9965\right)$. At the individual level, the two-cycle version of the model was preferred for 29/43 adults and 33/40 children (Table 1). These findings suggest that for the majority of participants and trials, estimates were made relative to both endpoints plus an inferred midpoint, rather than relative to the two endpoints alone.

The fits of the two-cycle proportional models to the estimation data shown in Figure 3 are good, and these models clearly capture some systematic qualities of these data that cannot be explained by a simple linear fit. However, the high $\mathrm{R}^{2}$ values for these fits are partly due to the linear increase of the estimated position with the target position. To explain systematic deviations from this linear increase, it is necessary to look at bias in the data by subtracting the target values from their corresponding estimates; when models are fit to data presented in this form, lower $\mathrm{R}^{2}$ values typically result. The resulting bias plots are shown in Figure 4 (left column), fit by the same pure two-cycle models seen in Figure 3. It is apparent that the two-cycle models cannot account for some asymmetries in participants' median estimation bias: the model

\footnotetext{
${ }^{4}$ Akaike Information Criterion, adjusted for small sample sizes, provides a measure of how well different models can explain data while taking differing numbers of parameters into account; Burnham, Anderson, \& Huyvaert, 2011; Burnham \& Anderson, 2002). $\triangle$ AICc refers to the difference in AICc values between another model and the preferred model, which has the lowest score. Burnham and Anderson (2002) proposed the following interpretation guidelines: "As a rough rule of thumb, models having a $\Delta$ within 12 of the [preferred] model have substantial support and should receive considerations in making inferences. Models having $\Delta$ within about 4-7 of the [preferred] model have considerably less support, while models with $\Delta>10$ have either essentially no support and might be omitted from further consideration or at least fail to explain some substantial structural variation in the data." (p. 446).
} 
underpredicts bias in some regions of the line and overpredicts in others $\left(\mathrm{R}^{2}=0.4878\right.$ for adults; $\mathrm{R}^{2}=0.4997$ for children).

To account for similar asymmetries observed previously, some researchers have proposed a mixed model: a weighted combination of the one-cycle and two-cycle versions of the CPM depicted in Figure 2A \& 2B (Hollands \& Dyre, 2000, see Eq. 9). The theoretical motivation for introducing such a mixed model is clear: the pure one-cycle model is expected to fit the data when estimates are made relative to two reference points. The pure two-cycle model is expected when estimates are made relative to those two reference points plus a midpoint. But all participants need not use the same reference point strategy, and individuals need not use the same strategy on every trial. In the likely event that multiple strategies contribute to participants' estimates, the mixed model is needed.

We applied the mixed model to the estimation bias data and compared it to the pure twocycle model. The pure model requires only one parameter $(\beta)$. The mixed model includes separate values of $\beta$ for each component ( $\beta_{1}$ for the one-cycle component; $\beta_{2}$ for the two-cycle component) and a weighting parameter that determines the relative contributions of the two components (taking values between 0 and 1; a higher value represents a larger contribution from the one-cycle component). The resulting improved fits are shown in Figure 4 (right column) for adults $\left(\mathrm{R}^{2}=0.9282\right)$ and children $\left(\mathrm{R}^{2}=0.9113\right)$, and resulting parameter values are consistent with those reported by Hollands \& Dyre (2000). AICc scores support the mixed model as a markedly better explanation of the bias data than the pure model for both age groups (for adults, $\Delta \mathrm{AICc}=$ 69.75; for children, $\Delta \mathrm{AICc}=60.86$ ), and the mixed model provided a better explanation than the individual's best pure model (1-cycle or 2-cycle) for 34/43 adults and 28/40 children (Table 2). 
Thus, the Cyclical Power Model (CPM) can provide a strong explanation of data that have previously been explained in terms of the Bayesian combination of multiple information sources described by the Category Adjustment model. The non-Bayesian CPM is appealing in part because it is simpler (requiring fewer parameters, for example) than the CA model.

Moreover, the CA model's predictions appear inconsistent with our data at the individual level. The CA model predicts that estimates should generally be adjusted toward the prototypical center of a spatial category, with the degree and direction of adjustment varying with the number of categories into which the observer subdivides the space. For example, some observers may treat the space in question as a single category, in which case estimates should generally be adjusted toward the center of the entire space (the prototypical center of the single spatial category). Alternatively, other observers may subdivide the space into two parts (using two spatial categories), in which case estimates within each category should generally be adjusted toward the center of that category. In this latter case of subdivision into two spatial categories, about half the estimates will (coincidentally) be displaced toward the overall center, and about half will (coincidentally) be displaced away from the overall center.

However, for many individuals in our study, most estimates are displaced away from the overall center of the line (at least $75 \%$ of estimates for 19/43 adults and 6/40 children; and in a few cases, nearly all estimates: see Figure 5). This is not predicted by the CA model whether a participant treats the space as a single category or subdivides it into two. The CPM, on the other hand, can accommodate these results through individual differences in the values of the model's parameters and strategic choices of reference points. Though further work is needed to explore this point, we do not believe the CA model can account for this result (see also Crawford, Landy, \& Presson, 2014, for prior findings of bias away from category centers that are inconsistent with 
the CA model's posited bias towards prototype values; see also the dynamic field theory, e.g. Spencer et al., 2012).

Conclusions. This work provides clear evidence that a relatively simple psychophysical model of estimation bias (the Cyclical Power Model) offers an excellent alternative explanation of adults' and children's performance in one domain of cognition in which complex Bayesian models are frequently invoked. The estimation biases that arise in these types of tasks therefore don't necessarily provide evidence of Bayesian cue combination. The CPM has theoretical links to prior work in perceptual psychology, and it has previously been shown to provide a good explanation of a wide variety of phenomena; the present work shows that children's and adults' spatial position estimation is among these. Further, this work provides preliminary evidence that the Category Adjustment model may be incompatible with individual performance patterns (for a prior case in which group data appeared to support a Bayesian account while individual data did not, see Mozer et al., 2008). The CPM also has limitations: for example, in the form presented here it does not speak to the well-established effects of delay time on estimation bias (e.g., Crawford et al., 2014; Hund \& Plumert, 2002). Nonetheless, this work highlights the broader need to consider non-Bayesian models of cognition in domains that are populated largely by Bayesian models. 


\section{References}

Barth, H. C., \& Paladino, A. M. (2011). The development of numerical estimation: Evidence against a representational shift. Developmental Science, 14, 125-135.

Booth, J. L., \& Siegler, R. S. (2006). Developmental and individual differences in pure numerical estimation. Developmental Psychology, 41, 189-201.

Booth, J. L., \& Siegler, R. S. (2008). Numerical magnitude representations influence arithmetic learning. Child Development, 79, 1016-1031.

Bowers, J. S., \& Davis, C. J. (2012). Bayesian just-so stories in psychology and neuroscience. Psychological Bulletin, 138, 389-414.

Burnham, K. P., \& Anderson, D. R. (2002). Model selection and multimodel inference. A practical information-theoretic approach (2nd ed). New York: Springer.

Burnham, K. P., \& Anderson, D. R., \& Huyvaert, K.P. (2011). AIC model selection and multimodel inference in behavioral ecology: some background, observations, and comparisons. Behavioral Ecology and Sociobiology, 65, 23-35.

Cicchini, G.M., Arreghi, R., Cecchetti, L., Giusti, M., \& Burr, D. (2012). Optimal encoding of interval timing in expert percussionists. Journal of Neuroscience, 32, 1056-1060.

Cohen, D. J., \& Blanc-Goldhammer, D. (2011). Numerical bias in bounded and unbounded number line tasks. Psychonomic Bulletin and Review, 18, 331-338.

Cohen, D. J., \& Sarnecka, B. (2014). Children's number-line estimation shows development of measurement skills (not number representations). Developmental Psychology, 50, 16401652.

Crawford, L.E., \& Duffy, S. (2012). Sequence effects in estimating spatial location. Psychonomic Bulletin \& Review, 17, 725-730. 
Crawford, L.E., Landy, D., \& Presson, A.N. (2014). Bias in spatial memory: Prototypes or relational categories? Proceedings of the Cognitive Science Society.

Duffy, S., Huttenlocher, J., \& Levine, S. (2005). It's all relative: How young children encode extent. Journal of Cognition and Development, 6, 51-63.

Duffy, S., Huttenlocher, J., \& Crawford, L. E. (2006). Children use categories to maximize estimation. Developmental Science, 9, 597-603.

Erlick, D.E. (1964). Absolute judgments of discrete quantities randomly distributed over time. Journal of Experimental Psychology, 67, 475-482.

Frick, A. \& Newcombe, N. (2012). Getting the big picture: Development of spatial scaling abilities. Cognitive Development, 27, 270-282.

Friedman, A., Montello, D.R. \& Burte, H. (2012). Location memory for dots in polygons versus cities in regions: Evaluating the category adjustment model. Journal of Experimental Psychology: Learning, Memory, and Cognition, 38, 1336-1352

Griffiths, T. L., Chater, N., Norris, D., \& Pouget, A. (2012). How the Bayesians got their beliefs (and what those beliefs actually are). Psychological Bulletin, 138, 415-422.

Hollands, J.G., \& Dyre, B.P. (2000). Bias in proportion judgments: The cyclical power model. Psychological Review, 107, 500-524.

Holden, M. P., Newcombe, N. S., \& Shipley, T. F. (2013). Location memory in the real world: Category adjustment effects in 3-dimensional space. Cognition, 128, 45-55.

Holden, M. P., Curby, K. M., Newcombe, N. S., \& Shipley, T. F. (2010). A category adjustment approach to memory for spatial location in natural scenes. Journal of Experimental Psychology: Learning, Memory, and Cognition, 36, 590-604. 
Hund, A., \& Plumert, J. (2003). Delay-induced bias in children's memory for location. Child Development, 73, 829-840.

Huttenlocher, J., Hedges, L. V., \& Duncan, S. (1991). Categories and particulars: Prototype effects in estimating spatial location. Psychological Review, 98, 352-368.

Huttenlocher, J., Hedges, L. V., \& Vevea, J. L. (2000). Why do categories affect stimulus judgment? Journal of Experimental Psychology: General, 129, 220-241.

Huttenlocher, J., \& Lourenco, S.F. (2007). Using spatial categories to reason about location. In J. Plumert \& J. Spencer (Eds.), The Emerging Spatial Mind (pp. 3-24). New York, NY: Oxford University Press.

Huttenlocher, J., Newcombe, N., \& Sandberg, E. H. (1994). The coding of spatial location in young children. Cognitive Psychology, 27, 115-147.

Huttenlocher, J., Newcombe, N., \& Vasilyeva, M. (1999). Spatial scaling in young children. Psychological Science, 107, 393-397.

Jazayeri, M., \& Shadlen, M.N. (2010). Temporal context calibrates interval timing. Nature Neuroscience, 13, 1020-1026.

Jones, M., \& Love, B. C. (2011). Bayesian fundamentalism or enlightenment? On the explanatory status and theoretical contributions of Bayesian models of cognition. Behavioral \& Brain Sciences, 34, 188-231.

Marcus, G. F., \& Davis, E. (2013). How robust are probabilistic models of higher-level cognition? Psychological Science, 24, 2351-2360.

Mozer, M. C., Pashler, H., \& Homaei, H. (2008). Optimal predictions in everyday cognition: The wisdom of individuals or crowds? Cognitive Science, 32, 1133-1147.

Nakajima, Y. (1987). A model of empty duration perception. Perception, 16, 485-520. 
Rouder, J., \& Geary, D. (in press). Children's cognitive representation of the mathematical number line. Developmental Science.

Schutte, A. R., Spencer, J. P., \& Schoner, G. (2003). Testing the dynamic field theory: Working memory for locations becomes more spatially precise over development. Child Development, 74, 1393-1417.

Shuford, E. H. (1961). Percentage estimation of proportion as a function of element type, exposure time, and task. Journal of Experimental Psychology, 61, 430-436.

Siegler, R. S., \& Booth, J. L. (2004). Development of numerical estimation in young children. Child Development, 75, 428-444.

Siegler, R. S., \& Opfer, J. E. (2003). The development of numerical estimation: evidence for multiple representations of numerical quantity. Psychological Science, 14, 237-250.

Slusser, E. B., Santiago, R. T., \& Barth, H. C. (2013). Developmental change in numerical estimation. Journal of Experimental Psychology: General, 142, 193-208.

Spence, I. (1990). Visual psychophysics of simple graphical elements. Journal of Experimental Psychology: Human Perception and Performance, 16, 683-692.

Spencer, J., Austin, A., \& Schutte, A.R. (2012). Contributions of dynamic systems theory to cognitive development. Cognitive Development, 27, 401-418.

Sullivan, J., Juhasz, B., Slattery, T., \& Barth, H. (2011). Adults’ number-line estimation strategies: Evidence from eye movements. Psychonomic Bulletin \& Review, 18, 557-563.

Teghtsoonian, R. (2012). The standard model for perceived magnitude: A framework for (almost) everything known about it. American Journal of Psychology, 125, 165-174.

Varey, C. A., Mellers, B. A., \& Birnbaum, M. H. (1990). Judgments of proportions. Journal of Experimental Psychology, 16, 613-625. 
Table 1

Children's and Adults' Preferred Models: 1-Cycle Versus 2-Cycle

\begin{tabular}{|c|c|c|c|}
\hline \multicolumn{2}{|c|}{ Children } & \multicolumn{2}{|c|}{$\underline{\text { Adults }}$} \\
\hline Preferred model & $\triangle \mathrm{AlCc}$ & Preferred model & $\Delta \mathrm{AlCc}$ \\
\hline 2-cycle & 8.896 & 2-cycle & 10.99 \\
\hline 2-cycle & 9.084 & 2-cycle & 13.15 \\
\hline 2-cycle & 8.113 & 2-cycle & 20.23 \\
\hline 2-cycle & 15.66 & 2-cycle & 31.07 \\
\hline 1-cycle & 20.97 & 2-cycle & 28.69 \\
\hline 2-cycle & 8.556 & 2-cycle & 9.943 \\
\hline 2-cycle & 10.27 & 1-cycle & 9.55 \\
\hline 2-cycle & 0.493 & 2-cycle & 27.21 \\
\hline 1-cycle & 0.405 & 2-cycle & 5.979 \\
\hline 2-cycle & 14.3 & 2-cycle & 9.891 \\
\hline 2-cycle & 14.13 & 2-cycle & 3.927 \\
\hline 1-cycle & 1.361 & 2-cycle & 7.355 \\
\hline 2-cycle & 12.47 & 1-cycle & 4.53 \\
\hline 2-cycle & 10.94 & 2-cycle & 18.23 \\
\hline 2-cycle & 6.698 & 2-cycle & 6.234 \\
\hline 2-cycle & 1.813 & 1-cycle & 7.245 \\
\hline 1-cycle & 4.966 & 1-cycle & 21.07 \\
\hline 2-cycle & 7.912 & 2-cycle & 8.259 \\
\hline 1-cycle & 4.366 & 2-cycle & 26.81 \\
\hline 2-cycle & 17.65 & 2-cycle & 9.794 \\
\hline 2-cycle & 7.628 & 1-cycle & 4.633 \\
\hline 2-cycle & 16.7 & 2-cycle & 1.999 \\
\hline 1-cycle & 12.95 & 2-cycle & 2.725 \\
\hline 2-cycle & 3.757 & 1-cycle & 0.1905 \\
\hline 2-cycle & 15.11 & 2-cycle & 11.4 \\
\hline 2-cycle & 2.952 & 1-cycle & 9.29 \\
\hline 2-cycle & 17.66 & 2-cycle & 35.58 \\
\hline 2-cycle & 4.484 & 2-cycle & 6.886 \\
\hline 2-cycle & 7.402 & 2-cycle & 1.278 \\
\hline 2-cycle & 3.259 & 2-cycle & 21.86 \\
\hline 2-cycle & 2.578 & 2-cycle & 4.621 \\
\hline 2-cycle & 8.069 & 1-cycle & 5.45 \\
\hline 2-cycle & 0.7252 & 1-cycle & 0.946 \\
\hline 2-cycle & 4.135 & 2-cycle & 14.62 \\
\hline 2-cycle & 2.338 & 2-cycle & 25.68 \\
\hline 2-cycle & 2.771 & 2-cycle & 10.15 \\
\hline 1-cycle & 3.422 & 2-cycle & 8.662 \\
\hline 2-cycle & 10.19 & 1-cycle & 7.291 \\
\hline 2-cycle & 8.842 & 1-cycle & 0.7652 \\
\hline \multirow[t]{4}{*}{ 2-cycle } & 4.509 & 1-cycle & 3.762 \\
\hline & & 1-cycle & 4.495 \\
\hline & & 2-cycle & 6.029 \\
\hline & & 1-cycle & 4.988 \\
\hline
\end{tabular}


Table 2

Children's and Adults' Preferred Models: Best Pure Model Versus Mixed Model

\begin{tabular}{|c|c|c|c|}
\hline \multicolumn{2}{|c|}{$\underline{\text { Children }}$} & \multicolumn{2}{|c|}{$\underline{\text { Adults }}$} \\
\hline Preferred model & $\Delta \mathrm{AlCc}$ & Preferred model & $\triangle \mathrm{AlCc}$ \\
\hline 2-cycle & 3.381 & mixed & 20.72 \\
\hline 2-cycle & 3.029 & 2-cycle & 2.91 \\
\hline mixed & 4.611 & mixed & 10.16 \\
\hline mixed & 16.24 & mixed & 0.3888 \\
\hline mixed & 10.74 & mixed & 15.74 \\
\hline mixed & 2.489 & mixed & 0.07256 \\
\hline mixed & 4.801 & 1-cycle & 0.4192 \\
\hline mixed & 4.885 & mixed & 4.072 \\
\hline mixed & 31.64 & mixed & 26.07 \\
\hline mixed & 13.67 & mixed & 32.9 \\
\hline 2-cycle & 3.402 & mixed & 49.63 \\
\hline mixed & 6.998 & mixed & 2.577 \\
\hline 2-cycle & 4.383 & mixed & 13.29 \\
\hline mixed & 4.536 & mixed & 7.357 \\
\hline mixed & 9.509 & 2-cycle & 1.294 \\
\hline mixed & 4.211 & mixed & 32.43 \\
\hline 1-cycle & 4.7 & mixed & 17.85 \\
\hline mixed & 6.746 & mixed & 9.059 \\
\hline mixed & 1.206 & mixed & 21.1 \\
\hline 2-cycle & 4.662 & mixed & 9.314 \\
\hline mixed & 9.222 & mixed & 42.08 \\
\hline mixed & 0.01239 & mixed & 32.52 \\
\hline mixed & 2.853 & mixed & 37.09 \\
\hline mixed & 2.723 & mixed & 60.41 \\
\hline mixed & 0.8618 & 2-cycle & 4.589 \\
\hline 2-cycle & 3.781 & mixed & 5.946 \\
\hline mixed & 10.05 & 2-cycle & 1.411 \\
\hline 2-cycle & 4.196 & mixed & 32.2 \\
\hline 2-cycle & 3.023 & mixed & 24.28 \\
\hline mixed & 5.175 & mixed & 5.332 \\
\hline mixed & 10.49 & mixed & 4.317 \\
\hline mixed & 24.62 & mixed & 8.628 \\
\hline mixed & 9.696 & mixed & 5.725 \\
\hline 2-cycle & 1.674 & mixed & 37.36 \\
\hline mixed & 15.22 & 2-cycle & 3.069 \\
\hline mixed & 3.78 & mixed & 34.91 \\
\hline 1-cycle & 4.785 & 2-cycle & 2.258 \\
\hline mixed & 5.23 & 1-cycle & 2.18 \\
\hline mixed & 1.515 & mixed & 44.94 \\
\hline \multirow[t]{4}{*}{ 2-cycle } & 1.643 & mixed & 7.818 \\
\hline & & mixed & 6.778 \\
\hline & & mixed & 21.78 \\
\hline & & 1-cycle & 4.587 \\
\hline
\end{tabular}




\section{Figure Captions}

Figure 1. Locations in which toddlers searched for a toy hidden in a long thin sandbox (to be reprinted pending permission from Elsevier), Huttenlocher, Newcombe, \& Sandberg, 1994.

Figure 2. Overview of proportion estimation model, showing predicted estimation patterns for 3 possible values of $\beta$. Figures $2 \mathrm{~A}$ and B depict one- and two-cycle versions of the model (the twocycle version depicts predicted estimation pattern for observers who use a middle reference point). These plots show example values of $\beta \leq 1$; an inverse pattern of over- and underestimation results if $\beta>1$.

Figure 3. Median spatial position estimates of adults (top) and 9-and 10-year-old children (bottom). The solid line represents the preferred model. The dashed line shows $y=x$. Note: no numerals were presented to participants during the spatial task; numerals are shown here to facilitate graph reading.

Figure 4. Estimation bias in adults (top) and children (bottom). Positive values indicate estimates shifted to the right of the target value on the response line; negative values are shifted to the left. The left column depicts fits of the pure 2-cycle proportion estimation model, which was preferred to the one-cycle; the right column depicts fits of the mixed model (weighted combination of the pure 1- and 2-cycle models), which was preferred to the pure 2-cycle. Figure 5. Bias in the estimates of an individual child participant. 
Figure 1.

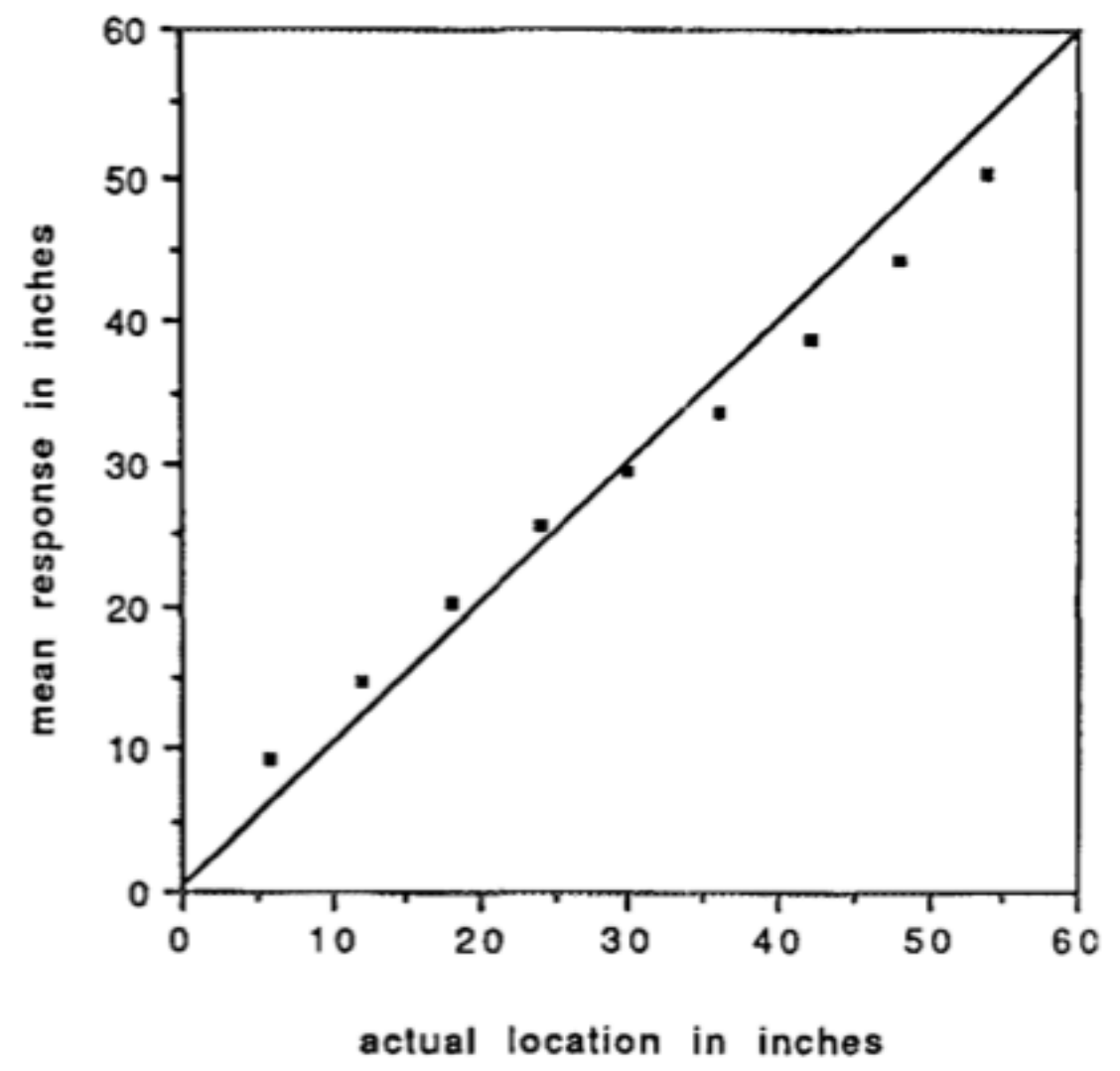

FIG. 2. Mean response locations for Experiment 1: 16- to 24-month-olds-sandbox. 
Figure 2.

Model predictions if observers use Model predictions if observers use 2 reference points ( 0 and 1$) \quad 3$ reference points $(0,1$, and a midpoint)
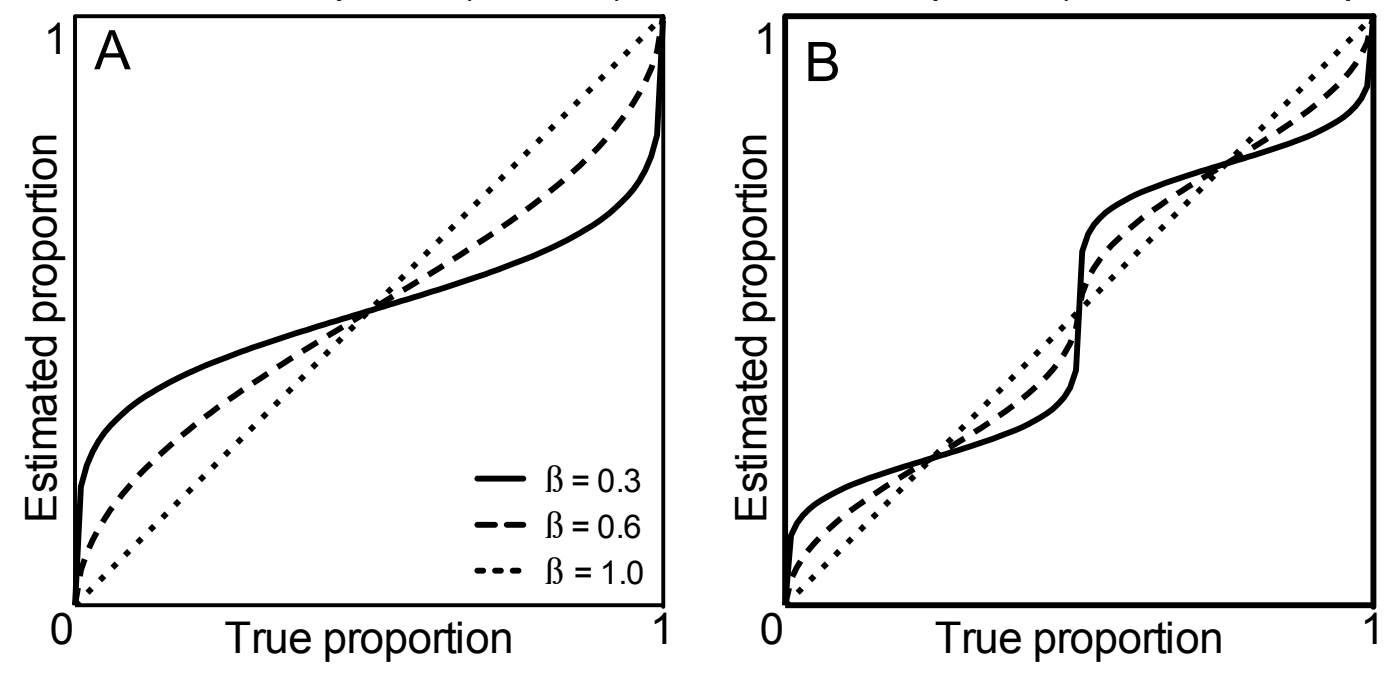
Figure 3.
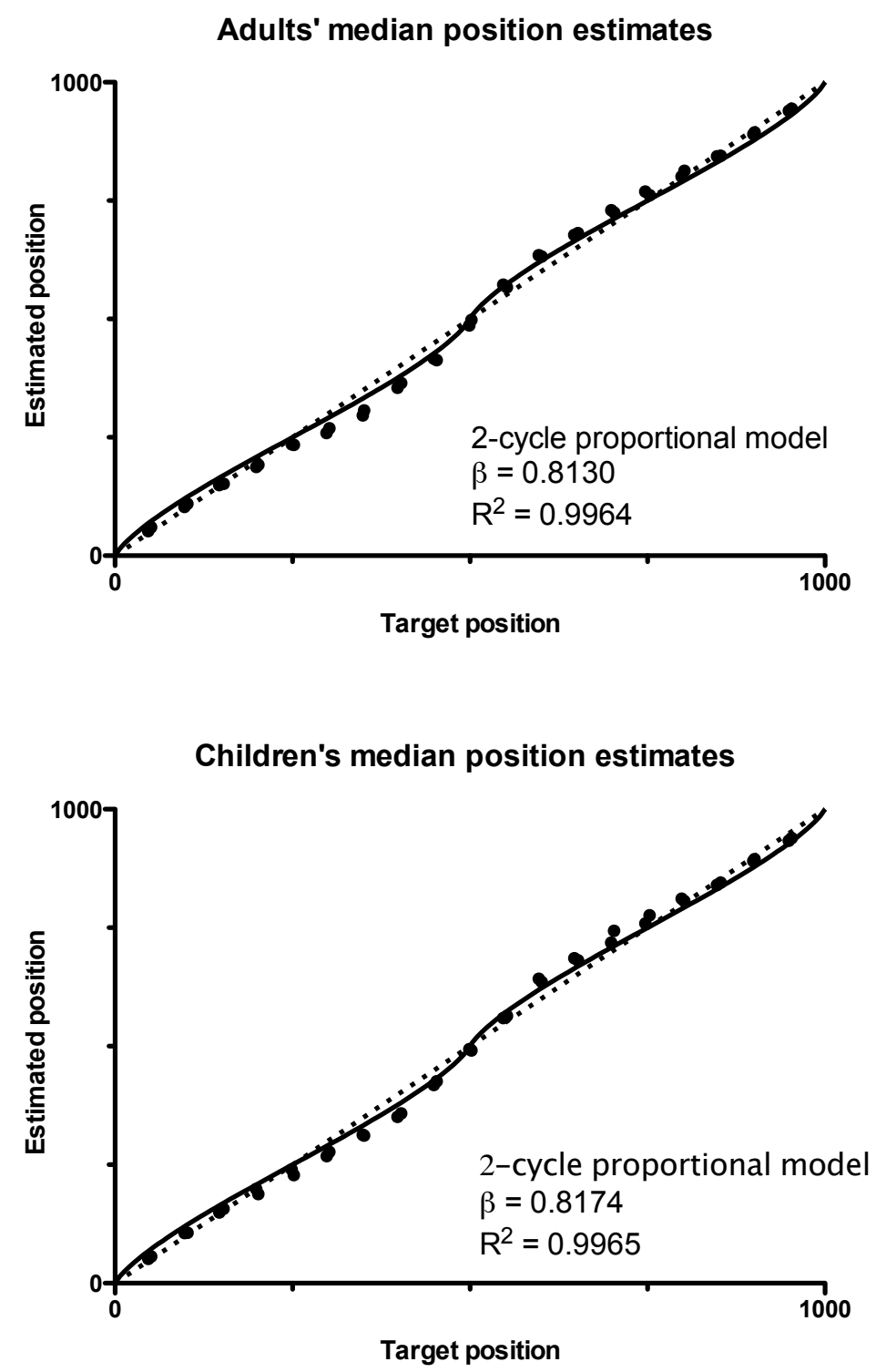
Figure 4.
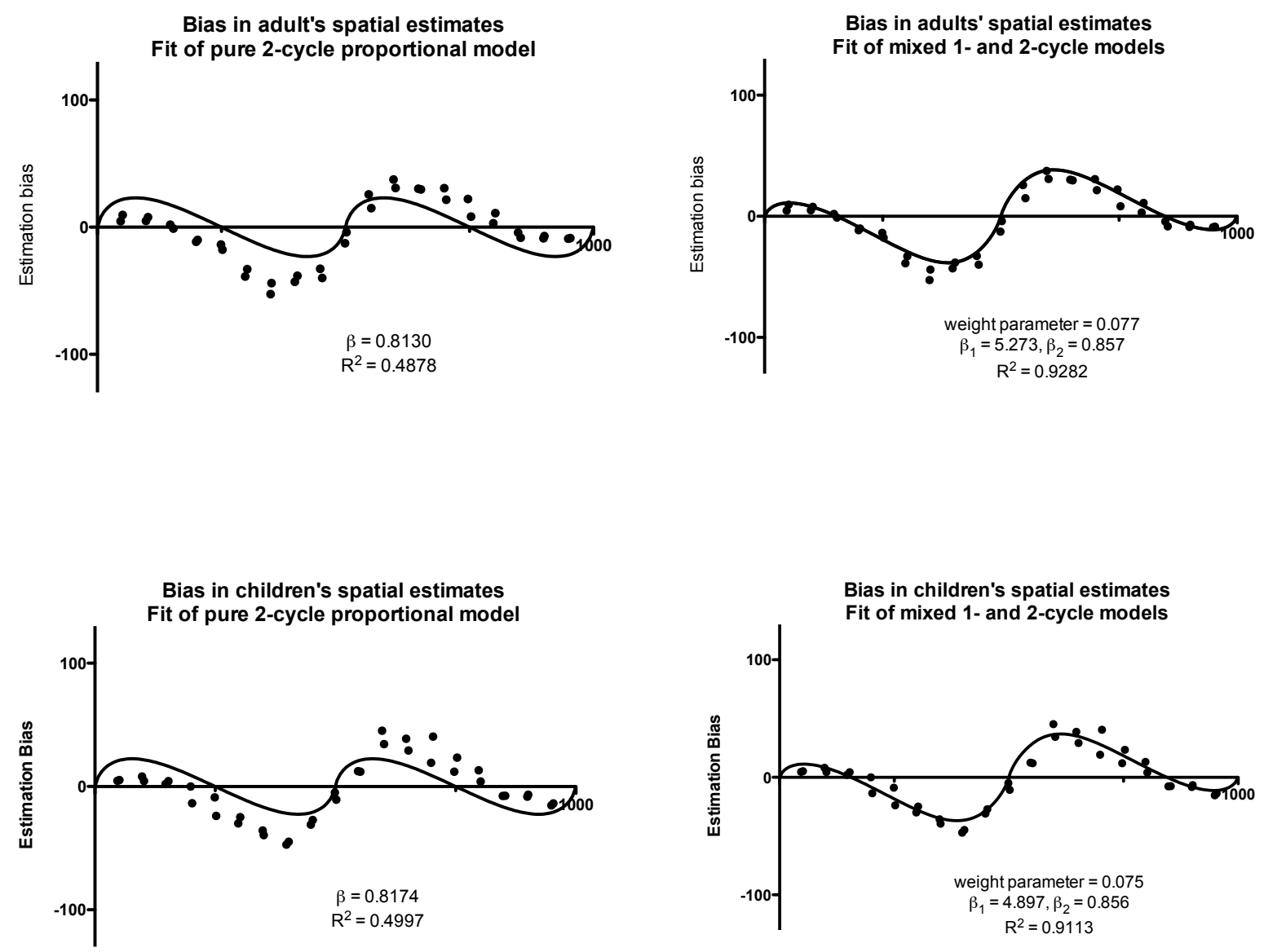
Figure 5.

Bias in an individual child's spatial estimates

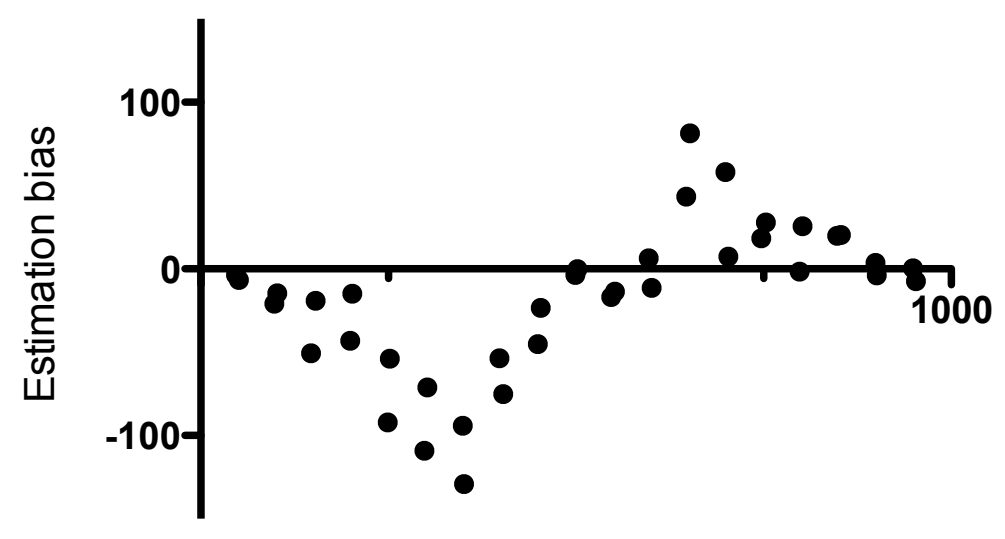

